\title{
La performatività posthuman
}

\author{
Federica Patti \\ LaRete Art Project
}

\begin{abstract}
L'accelerazione tecnologica continua a rivoluzionare la liveness lasciando emergere un alto gradiente di performatività anche nelle semplici abitudini multimediali quotidiane. L'ubiquità pervasiva dei media digitali influenza profondamente i modi e gli stili in cui la performance viene agita e fruita. Attivando efficaci dinamiche di connessione e coinvolgimento del pubblico, strumenti come lo streaming, la realtà virtuale e aumentata, l'intelligenza artificiale, il mapping, l'interaction design consentono oggi di allestire esperienze multimediali che offrono una dimensione sociale e comunicativa completamente diversa, all'insegna della transdisciplinarietà e dell'approccio post-antropocentrico. La live media performance è da considerarsi quindi come specifico settore di produzione artistica e di indagine, in cui è possibile far rientrare diverse tipologie di formati e pratiche. Gli agenti elettronici in scena vengono progettati per trasmettere un senso di interconnessione profonda tra i protagonisti, il pubblico e tutto ciò che li circonda. Il palcoscenico diventa così una dimensione sospesa, un "luogo della mente" sinestetico e immersivo, in cui viene ricostruita la realtà come risultato di un agire non soltanto umano. La tecnosfera diviene in questo senso un partner di lavoro. Attraverso l'analisi di ricerche e di linguaggi artistici multimediali performativi emblematici di questo settore disciplinare, si proporrà una suggestione interpretativa transdisciplinare post-antropocentrica e postumana, prestando attenzione alle forme sia umane sia non umane di azione e gesto espressivo.
\end{abstract}

The technological acceleration is constantly revolutionizing liveness, allowing a high degree of performativity to emerge even in simple everyday multimedia habits. The pervasive ubiquity of digital media profoundly influences how performance is delivered and experienced. By activating effective dynamics of audience involvement, tools such as live streaming, virtual and augmented reality, artificial intelligence, mapping and interaction design now make it possible to create multimedia experiences that offer a whole social and communicative dimension, characterized by a transdisciplinary and post-anthropocentric approach. Therefore, live media performance should be considered a specific field of artistic production and investigation, in which different formats and practices can be included. The electronic agents on stage are designed to convey a sense of deep interconnection between the performers, the audience and everything around them. The scene thus becomes a suspended dimension, a synaesthetic and immersive "place of the mind" in which reality is reconstructed as the result of not-just-human action. In this sense, the technosphere becomes a working partner. Through the analysis of key research and artistic experiences of this disciplinary field, a transdisciplinary post-anthropocentric and post-human interpretative perspective will be proposed, focusing on both human and non-human forms of action and expressive gesture.

\section{Parole chiave/Key Words}

Metaverso; gamification; postumano; intelligenza artificiale.

Metaverse; gamification; posthuman; Al. 


\title{
Human, Non-human, Post-human
}

L'accelerazione tecnologica continua a rivoluzionare il concetto di liveness (de visu) lasciando emergere la possibilità di un alto gradiente di performatività anche nelle semplici abitudini multimediali quotidiane ${ }^{1}$. Le interrelazioni esistenti fra corpi, software e macchine, la simbiosi fra utenti e devices, onnipresenti e interconnessi, permettono una esibizione individuale pervasiva, e annullano la percezione delle differenze fra agire umano e non. La concezione postumana del rapporto, anche fisico, uomo-macchina qui proposta mutua l'approccio elaborato da Rosi Braidotti: attraverso la rilettura materialista femminista (e post antropocentrica) del pensiero filosofico, la declinazione del concetto di postumano promossa da Braidotti mira infatti a scardinare ogni tipo di dicotomia tra mente e corpo, culturale, naturale e tecnologico, addirittura tra vita e morte; con lo scopo finale di produrre una rinnovata eticità, capace di creare «attivamente orizzonti sociali di speranza», oltre ogni discriminazione.

\begin{abstract}
All'inizio di tutto c'è Lui: I'ideale classico di "Uomo", formulato prima da Protagora come "la misura di tutte le cose", poi rinnovato nel Rinascimento italiano come modello universale e rappresentato nell'Uomo Vitruviano di Leonardo da Vinci. Un ideale di perfezione corporea che, secondo il detto classico mens sana in corpore sano, si raddoppia come insieme di valori mentali, discorsivi e spirituali. [...] Questo paradigma eurocentrico implica la dialettica del sé e dell'altro e la logica binaria dell'identità e dell'alterità come motore e logica culturale dell'umanesimo universale. La soggettività è equiparata alla coscienza, alla razionalità universale e al comportamento etico autoregolato, mentre I'alterità è definita come la sua controparte negativa e speculare. [...] II mio punto è che questo approccio, che si basa sull'opposizione binaria tra il dato e il costruito, è attualmente sostituito da una comprensione non dualistica dell'interazione naturacultura. A mio avviso quest'ultima è associata e sostenuta da una filosofia monistica, che rifiuta il dualismo, specialmente l'opposizione natura-cultura e sottolinea invece la forza auto-organizzativa (o auto-poietica) della materia vivente. I confini tra le categorie del naturale e del culturale sono stati spostati e in gran parte offuscati dagli effetti del progresso scientifico e tecnologico. Questo continuum natura-cultura è il punto di partenza condiviso per il mio approccio alla teoria postumana. Se questo presupposto post-naturalistico si traduca successivamente in sperimentazioni giocose con i confini della perfettibilità del corpo, in panico morale per lo sconvolgimento di credenze secolari sulla "natura" umana o nella ricerca sfruttatrice e orientata al profitto del capitale genetico e neurale, resta comunque da vedere. ${ }^{2}$
\end{abstract}

\footnotetext{
${ }^{1}$ P. Auslander, At the Listening Post, or, do machines perform?, in «International Journal of Performance Arts and Digital Media» vol. 1, n. 1, 2005, pp. 5-10.

2 R. Braidotti, The Posthuman, Polity Press, Cambridge 2013, p. 13 [traduzione mia]; su postumanesimo e sulla filosofia femminista post-antropocentrica cfr. D. Haraway, Manifesto Cyborg. Donne, tecnologie e biopolitiche del corpo, Feltrinelli, Milano 2018; R. Peppeler, The Posthuman Condition: Consciousness Beyond the Brain, In-
} 
Mantenendo questa stessa apertura interpretativa, Karen Barad propone una metafisica postumana e performativa, in cui il mondo è un processo aperto di materia in evoluzione, attraverso il quale essa stessa importa, acquisisce significato e forma nella realizzazione di diverse possibilità. II Realismo agenziale ${ }^{3}$ della Barad definisce il processo con il quale la realtà si materializza dinamicamente in modo aperto e continuo, attraverso i gesti, le relazioni, le intra-azioni di fattori umani e non umani a livello atomico, producendo fenomeni e dispositivi. Barad cita il fisico-filosofo Niels Bohr, per il quale le cose non hanno confini o proprietà intrinsecamente determinate, il mondo non è fatto di oggetti chiusi ma di dinamiche sempre attive. Questa rottura di Bohr con Newton, Cartesio e Democrito non è basata su "mera riflessione filosofica oziosa" ma su nuove scoperte empiriche della fisica atomica che vennero alla luce durante il primo quarto del ventesimo secolo; secondo Bohr, l'unità epistemologica primaria non è l'oggetto indipendente con confini e proprietà intrinseci ma piuttosto i fenomeni.

Nelle sue pubblicazioni divulgative, Barad allora trae dagli esperimenti sui quanti di Bohr un modello valido, queer, per una nuova narrazione dinamica della realtà, attenta e aperta alle differenze, grazie all'intreccio postulato fra materia e significato ${ }^{4}$.

La suggestione di centralità dell'azione stessa come gesto di definizione identitaria ( / play Therefore I $\mathrm{Am}^{5}$ ) così proposta da Barad invita ad un riposizionamento radicale del punto di vista critico, verso le seguenti considerazioni: le intra-azioni che individua sono dialoghi, relazioni fisiche della materia tutta, descrivibili come danza fra entità atomiche.

tellect, Bristol 2003; E. Povinelli, The Ends of Humans: Anthropocene, Autonomism, Antagonism, and the Illusions of Our Epoch, in «The South Atlantic Quarterly», vol. 116, n. 2, 2017, pp. 293-310.

3 "Usare le scienze per ripensare le scienze umane": a partire dagli anni Duemila, Barad propone una mutazione accademica verso l'alfabetizzazione agenziale, tramite pedagogie dove la scienza viene insegnata e recepita in modo transdisciplinare e responsabile, intrecciandosi con le scienze umane, tenendo ben presente l'entanglement, cioè che scienza e società costituiscono l'un l'altra. II suo "realismo agenziale" rappresenta il processo con il quale, su base fisica, la realtà si materializza dinamicamente attraverso le intra-azioni similcellulari di fattori umani e non umani, producendo fenomeni e dispositivi.

${ }^{4}$ Per un'introduzione al pensiero di Barad cfr. K. Barad, Re(con)figuring Space, Time, and Matter, in M. DeKoven (a cura di) Feminist Locations: Global and Local, Theory and Practice, Rutgers University Press, New Brunswick, 2001; K. Barad, Meeting the Universe Halfway: Quantum Physics and the Entanglement of Matter and Meaning, Duke University Press, Durham 2007; K. Barad, What is the Measure of Nothingness? Infinity, Virtuality, Justice / Was ist das Maß des Nichts? Unendlichkeit, Virtualität, Gerechtigkeit, in dOCUMENTA (13): 100 Notes - 100 Thoughts / 100 Notizen - 100 Gedanken, Hatje Cantz Verlag, Ostfildern 2012; K. Barad, On Touching -- The Inhuman That Therefore I Am, in «Differences: A Journal of Feminist Cultural Studies», vol. 23, n. 3, 2012, pp. 206-223.

${ }^{5}$ G.A. Voorhees, I Play Therefore I Am: Sid Meier's Civilization, Turn-Based Strategy Games and the Cogito, in «Games and Culture», vol. 4, n. 3, 2009, pp. 254-275. 
In questo senso, un approccio incentrato sulla performatività delle entità non prevede gerarchie né distinzioni: qualsiasi forma di intra-azione fra atomi della materia può essere classificata come atto performativo.

Le composizioni e le presentazioni stesse di Barad sono concepite come intra-azioni, ovvero come operazioni atte ad aprire la relazione e l'entanglement fra oratore e pubblico. Usa inoltre la diffrazione come fondamento del suo metodo di interpretazione scientifica, epistemologica, storica e letteraria, operando il salto che porta dalla meccanica quantistica all'epistemologia e alla antropologia sociale: secondo Barad non si tratta di «fare analogie tra il mondo degli atomi e il mondo sociale», piuttosto di affermare che «la fisica quantistica parla del mondo dentro ogni momento, della bomba dentro l'atomo ${ }^{6}$. La materia, tutta, non è oggettuale e chiusa ma dinamica, agisce e si trasforma attraverso l'entanglement, la relazione inseparabile - o «perturbante azione a distanza» come la definì Einstein.

In summary, the universe is agential intra-activity in its becoming. The primary ontological units are not "things" but phenomena-dynamic topological reconfigurings / entanglements / relationalities / (re)articulations. And the primary semantic units are not "words" but material-discursive practices through which boundaries are constituted. This dynamism is agency. Agency is not an attribute but the ongoing reconfigurings of the world. On the basis of this performative metaphysics, in the next section I propose a posthumanist refiguration of the nature of materiality and discursivity and the relationship between them, and a posthumanist account of performativity. ${ }^{7}$

Prendendo spunto in maniera metaforica da questa "rivoluzione prospettica", mutuando lo stile divulgativo-emotivo di Barad e suggerendo una chiave di lettura poetica altra, non empirica bensì intuitiva, questo articolo si propone di sottolineare le peculiarità della creazione e della fruizione di una esperienza performativa multimediale dal vivo,

\footnotetext{
${ }^{6}$ L. Borghi, Premessa, in K. Barad, Performatività della natura. Quanto e queer, Edizioni ETS, Pisa 2017, p. 8; su questa relazione fra discipline e sguardi Barad assume la "diffrazione" come approccio: "Basandomi su un suggerimento di Donna Haraway, quello che propongo è la pratica della diffrazione, della lettura diffrattiva per modelli di differenze che fanno la differenza. E intendo questo non come nozione additiva opposta alla sottrazione, piuttosto che essa è suggestiva, creativa e visionaria. La diffrazione, intesa con la fisica quantistica, non è solo una questione di interferenza, ma di intreccio [entanglement], una questione etico-onto-epistemologica. Questa differenza è molto importante: sottolinea il fatto che la conoscenza è un impegno materiale diretto, un taglio, dove i tagli fanno violenza ma aprono e rielaborano anche le condizioni agenziali della possibilità. Non $c^{\prime}$ è questo sapere a distanza. Invece di una separazione tra soggetto e oggetto, c'è un intreccio tra soggetto e oggetto, che si chiama "fenomeno" ", da K. Barad, La materia sente, conversa, soffre, desidera, anela e ricorda Intervista a Karen Barad, da: https://www.sovrapposizioni.com/blog/la-materia-sente-conversa-soffredesidera-anela-e-ricorda-intervista-a-karen-barad-prima-parte (ultimo accesso 10/11/2021).

${ }^{7}$ K. Barad, Posthumanist Performativity: Toward an Understanding of How Matter Comes to Matter, in «Signs», vol. 28, n. 3, 2003, p. 818.
} 
per articolare la ricerca necessaria ad affrontare l'algoritmica condizione umana nella complessità del presente. In primis, evidenziando come le funzionalità intrinseche proprie dei media inducano la relazione fra umano e non umano, l'esibizione e l'espressione di sé come azione generatrice di un gesto artistico anche al di fuori dei formati e dei contesti tradizionali, al di là della esplicita intenzione artistica, creando così un'opportunità di ribaltamento del flusso creativo, rendendo attivo e propositivo lo spettatore, che diviene autore radicale dei contenuti: grazie alla relazione con il complesso sistema tecnologico, il gesto creativo e performativo può trovare dimora nelle più semplici azioni quotidiane - dall'invio di un videomessaggio all'attraversamento di una piazza "sensibile".

Le culture digitali sono culture performative. La performatività nelle sue dimensioni eterogenee non può permettersi di ignorare le forze e gli effetti agenziali delle tecnologie digitali e dei loro legami con i corpi umani. [...] Fare ricerca e intervenire nel presente e nella performatività delle culture digitali richiede sensibilità e eterogeneità comuni a quelle delle Performance studies. Per fare ciò, tuttavia, il campo di studio è invitato ad abbracciare e confrontarsi più pienamente con la condizione tecnologica odierna e le performance umane / non umane o forse "postumane" che modellano i processi sociali e culturali. ${ }^{8}$

L'attenzione di questa lettura critica si concentrerà quindi sulla qualità dell'esperienza attivata, proposta e recepita dal sistema tecnologico, sul progetto artistico e sulla collocazione dell'opera, ponendo in grande rilevanza il tipo di interazione stabilita con l'utente. Dati questi elementi, diviene utile e possibile far rientrare in questo settore artistico allora anche dinamiche apparentemente extra artistiche, quali per esempio: le esperienze gaming, l'interactive storytelling, il live streaming, la data analysis (in particolare l'analisi del sentiment), l'addestramento di una rete neurale.

Complessi ecosistemi naturali, artificiali, sintetici e il reciproco intreccio relazionale diventano co-protagonisti del passato, del presente e del futuro della produzione e della

\footnotetext{
${ }^{8}$ T. Beyes, M. Leeker, I. Schipper, Performing the Digital. Performance Studies and Performances in Digital Cultures, transcript publishing, 2016, p. 7, [traduzione mia]. Per una ricognizione sul rapporto fra digitale e performance cfr. S. Dixon, Digital Performance: A History of New Media in Theater, Dance, Performance Art, and Installation, MIT Press, Cambridge 2007; S. Bay-Cheng, C. Kattenbelt, A. Lavender, Mapping Intermediality in Performance, Amsterdam University Press, Amsterdam 2010; C. Salter, Entangled: Technology and the Transformation of Performance, MIT Press, Cambridge 2010; P. Eckersall, H. Grehan, E. Scheer, New Media Dramaturgy. Performance, Media and New-Materialism, Palgrave Macmillan, Londra 2017.
} 
sperimentazione creativa e culturale, in linea con l'idea di performatività propria dello sguardo postumano di Barad:

[...] Propongo un'interpretazione squisitamente postumanista della performatività nozione che incorpora importanti fattori materiali e discorsivi, sociali e scientifici, umani e non umani, naturali e culturali. L'approccio postumanista chiama in causa il dato delle categorie differenziali quali "umano" e "non umano", esaminando le pratiche attraverso le quali questi confini e differenze vengono stabilizzate e destabilizzate: I'opus accademico di Donna Haraway - dai primati ai cyborg alle specie compagne - incarna questo punto. ${ }^{9}$

\section{Viva Arte Viva}

Walter Benjamin ha sostenuto come «ogni forma d'arte raggiunge livelli ed effetti che potrebbero essere pienamente ottenuti soltanto con un mutato standard tecnico, cioè una nuova forma $d^{\prime}{\text { arte }{ }^{10}}^{10}$ I molteplici linguaggi delle arti elettroniche hanno assorbito tutte le soluzioni formali novecentesche per riproporle nella videoinstallazione, nell'animazione grafica e nei live media, combinate al suono e dotate di movimento ${ }^{11}$. Possiamo quindi considerare i capolavori multimediali contemporanei come l'apice di un percorso, una summa che raggiunge una potenza espressiva mai vista prima e che apre a scenari inesplorati; percorsi che, al tempo stesso, fondano le proprie basi nelle sperimentazioni visionarie, pionieristiche, antesignane delle avanguardie storiche, e nella rivoluzione sociale, culturale, estetica di cui sono state promotrici ed emblemi.

Nella live media performance - o spettacolo multimediale dal vivo -, esprime (sin dalla definizione) nel medium, nella sua funzionalità, esecuzione e fruizione la liveness dell'opera/operazione artistica, quale sistema complesso in atto in cui le tecnologie svolgono un ruolo da protagonista. La live media performance è quindi da considerarsi allora come specifico settore di produzione artistica e di indagine, in cui è possibile far rientrare

\footnotetext{
${ }^{9}$ K. Barad, op. cit, p. 810.

${ }^{10}$ W. Benjamin, Aura e choc. Saggi sulla teoria dei media, Einaudi, Torino 2012, p. 68.

11 Sul rapporto fra forme estetiche, media e tecnologie cfr. J. Bolter, R. Grusin, Remediation: Understanding New Media, MIT Press, Cambridge 1998; N. Bourriaud, Estetica Relazionale, Postmediabooks, Milano $1998 ;$ M. Costa, L'estetica dei media. Avanguardie e tecnologia, Castelvecchi, Roma 1999; L. Manovich, II Linguaggio dei Nuovi Media, Edizioni Olivares, Milano 2002; A. Balzola, A.M. Monteverdi, Le arti multimediali digitali. Storia, tecniche, linguaggi, etiche ed estetiche del nuovo millennio, Garzanti, Milano 2004; S. O'Reilly, Il corpo nell'arte contemporanea, Einaudi, Torino 2009; M. Perniola, L'estetica contemporanea, il Mulino, Bologna 2011; C. Paul, Digital Art (3rd ed.), Thames \& Hudson, Londra 2015; G. Matteucci, Elementi per una estetica del contemporaneo, Bononia University Press, Bologna 2018.
} 
diverse tipologie di formati e pratiche, accomunate da peculiari elementi quali: multimedialità; interazione attiva uomo - macchina/software; esibizione/presentazione/azione e fruizione in diretta, dal vivo (nella nuova concezione del termine); stimolazione e coinvolgimento multisensoriale; progettazione site specific e community specific. Attivando efficaci dinamiche di connessione e coinvolgimento del pubblico, strumenti come i sistemi computazionali, gli algoritmi complessi e l'intelligenza artificiale consentono oggi di allestire esperienze multimediali che offrono una dimensione sociale e comunicativa completamente diversa, in cui il rapporto tra presenza fisica e virtualità, racconto, visione e interattività, linearità e combinazione di scelte rappresenta il tema dominante attorno cui ruotano la composizione, la fruizione e i contenuti ${ }^{12}$. Questi tools consentono così inedite e libere modalità di liveness e impongono la necessità di una nuova lettura storica e critica delle pratiche artistiche ad esse connesse, all'insegna della transdisciplinarietà e dell'approccio post antropocentrico.

Leggere una live media performance significa collocarla nel contesto di fruizione per cui è stata pensata - sia esso in presenza fisica dello spettatore, o da remoto - e comprendere le ragioni estetiche e drammaturgiche che hanno determinato la scelta di una precisa tecnologia ${ }^{13}$. Significa inoltre inseguirne le apparizioni, riconoscerne le nuove forme che spesso trascendono le classificazioni disciplinari, e intuirne proattivamente le evoluzioni, assumendo un atteggiamento critico empatico con le dinamiche mutevoli e sorprendenti della materia viva, in continua crescita e trasformazione. Attraverso la lettura interpretativa di alcuni esempi artistici di spettacolo dal vivo multimediale che si propone di seguito, è possibile sottolineare e individuare le peculiarità della creazione e della fruizione di una esperienza performativa multimediale dal vivo. Dati questi elementi, diviene utile e possibile far rientrare nel settore delle live media performance allora anche dinamiche apparentemente extra artistiche, individuandone via via diversi gra-

\footnotetext{
12 Sulla relazione tra arte, media e culture digitali cfr. D. Quaranta, Beyond New Media Art, Link Editions, Brescia 2013; F.J. Ricardo, The Engagement Aesthetic. Experiencing New Media Art through Critique, Bloomsbury Academic, Londra 2013; L. Cornell, E. Halter (a cura di), Mass Effect: Art and the Internet in the Twenty-First Century, MIT Press, Cambridge 2015; H. Steyerl, Duty Free Art: Art in the Age of Planetary Civil War, Verso books, Londra 2017; K. Kard (a cura di), Alpha Plus: anthology of digital art, Editorial Vortex, 2017; J. Bridle, Nuova Era Oscura, Nero, Roma 2019.

${ }^{13}$ A.M. Monteverdi, Leggere uno spettacolo multimediale. La nuova scena tra video mapping, interaction design e Intelligenza Artificiale, Dino Audino Editore, Roma 2020.
} 
dienti di liveness e delineandone la definizione e l'analisi transdisciplinare dei linguaggi e delle estetiche, nella vasta area di contaminazione fra informatica, neuroscienze, media, musica, performing arts e arti visive - seguendo le interpretative precedentemente proposte e un approccio decoloniale e postumano alla stessa analisi critica.

\section{«Viversi come spettacolo»}

Gli elementi, gli agenti elettronici in scena - composti da materiale prevalentemente software, video e audio - in una live media performance vengono progettati per trasmettere un senso di interconnessione profonda tra i protagonisti, il pubblico e tutto ciò che li circonda. L'interazione diretta tra i gesti coreografici e questi elementi rappresenta uno dei punti focali delle composizioni ipermediali dal vivo, capace di trasmettere un forte senso di unione e sincronicità tra la componente tecnologica e quella corporea umana, comunemente detta. Creando una temporanea bolla di simultaneità, uno spettacolo di questo tipo propone allo spettatore una "vertigine" visiva, spaziale, sensoriale e tecnologica, possibile solo grazie alla fortissima compenetrazione fra il mondo fisico e la sua amplificazione tecnica. Il palcoscenico diventa così una dimensione sospesa in cui è possibile perdere la cognizione dello spazio e del tempo, un "luogo della mente", sinestetico e immersivo, in cui viene ricostruita la realtà come risultato delle proprie stesse azioni, dove ogni gesto, di chiunque, ha una conseguenza immediata, ipermediata e precisa. Fondamentale in questo percorso diviene l'ascolto della tecnosfera intesa come vero e proprio partner di lavoro: gli schermi, i sensori, gli algoritmi, i circuiti meccanici, gli speaker diventano elementi attivi e reagenti ai movimenti, proprio come farebbe un performer in scena. O addirittura registi, agenti senzienti, indicatori di una drammaturgia possibile. E allora il corpo è tutto: i novantuno spot luminosi rotanti e danzanti nella sinfonia The Enlightenment del Quiet Ensemble, gli stormi di droni del duo olandese Studio Drift, le braccia soniche di Ultravioletto e gli assistenti robotici prodotti dalla Boston Dynamics.

$\angle D M B>$, algoritmo di intelligenza artificiale regista delle luci e dei suoni della trilogia Humane Methods del collettivo Fronte Vacuo (Marco Donnarumma, Margherita Pevere e Andrea Familari) è una entità viva in scena, che interagisce con il palcoscenico, i 
performers e il pubblico in tempo reale. Quale rete neurale allenata a processare i dati biometrici, ambientali, sonori e luminosi - provenienti dalle altre entità presenti nell'ambiente circostante, e di farne restituzione immediata e reattiva attraverso una ridistribuzione degli stimoli di luce e suoni nello spazio tempo dello spettacolo.

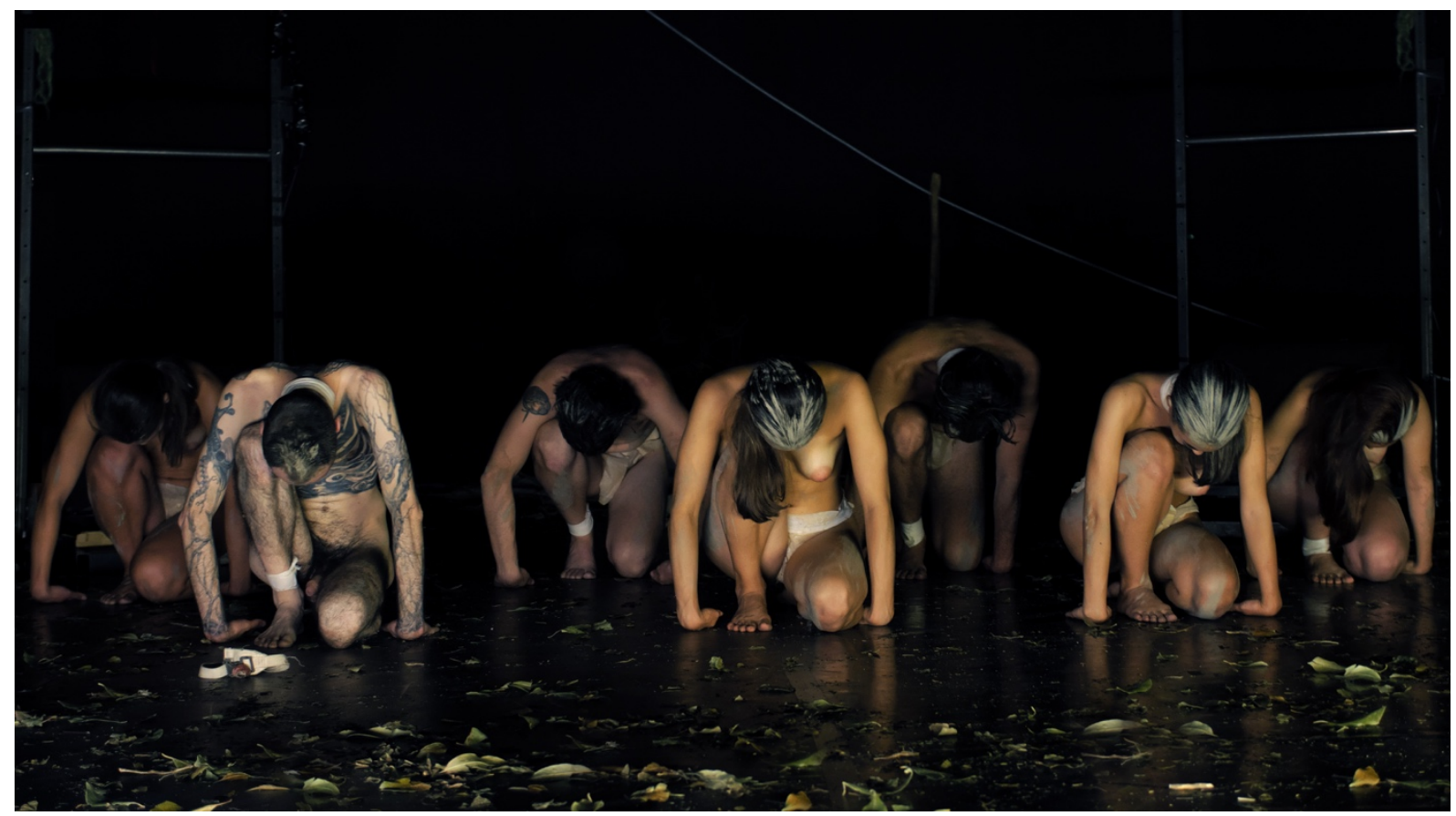

Fig. 01. Fronte Vacuo (Donnarumma, Familari, Pevere), Humane Methods, 2019. Foto: Piero Tauro

In Dökk, spettacolo di fuse*, la danza aerea di Elena Annovi duetta in tempo reale con un apparato tecnologico immersivo, pervasivo, interattivo, entangled, capace di stimolare una profonda empatia, generata dalla sincronicità e imprevedibilità dell'esistenza umana. Per ottenere questo risultato è stato sviluppato un sistema complesso unico, capace di presentare sul palco elaborate composizioni audio video, risultato della stretta interazione tra diversi dati generati in tempo reale: I'analisi del suono, il movimento della performer, il suo battito cardiaco, mappe interstellari e l'analisi sentimentale dei contenuti condivisi sui social network. La combinazione di queste informazioni rende ogni messa in scena unica, sempre diversa perché frutto della casualità e dell'imprevedibilità tipica della liveness multimediale interconnessa, sulla base delle informazioni analizzate in tempo reale. 


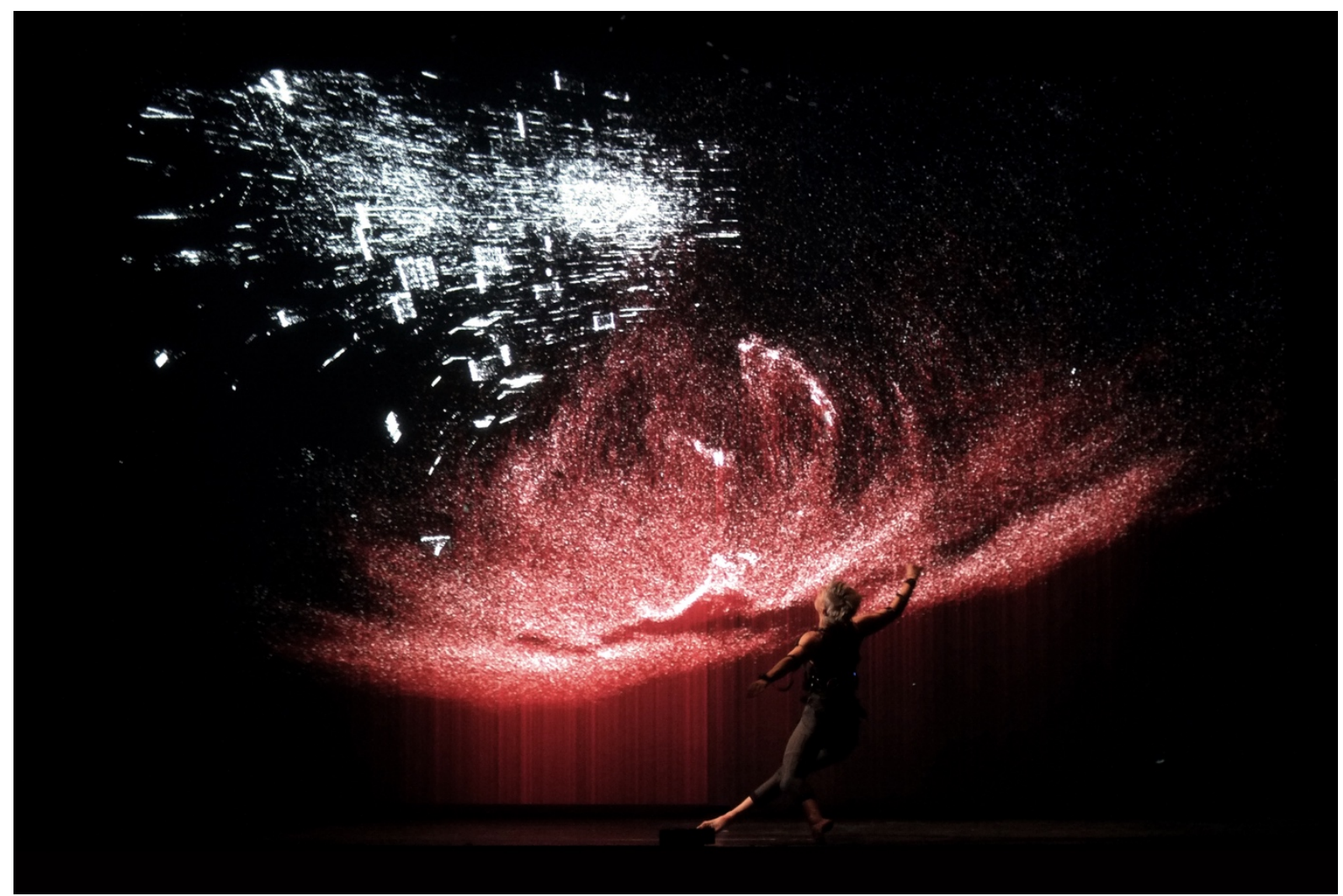

Fig. 02. fuse*, Dökk, 2017. Foto: Enrico Maria Bertani ed Emmanuele Coltellacci

Dökk è allora emblema della visione suggerita da Barad: dalle galassie alle molecole, tutto ciò che ci circonda non è che un insieme di atomi, particelle e campi elettromagnetici in vibrazione senza alcun significato apparente. Quando questi impulsi vengono interpretati dalla mente umana diventano colori, musica, ricordi ed emozioni: le fondamenta di ciò che ognuno percepisce come realtà. L'idea di ritmo e di danza viene spontanea alla mente quando si cerca di immaginare il flusso di energia che si trasmette attraverso le configurazioni che costituiscono il mondo delle particelle. La fisica contemporanea ci ha mostrato che movimento e ritmo sono proprietà essenziali delle particelle, che tutta la materia, sia sulla Terra sia nello spazio esterno, è coinvolta in una continua coreografia cosmica. I mistici orientali hanno una visione dinamica dell'Universo simile a quella della scienza odierna, e di conseguenza non sorprende il comune utilizzo dell'immagine del ballo per comunicare la loro intuizione della natura. La metafisica della danza cosmica ha trovato nell'Induismo la più profonda e splendida espressione nell'immagine di Shiva Nataraja danzante. Secondo la dottrina indù, tutta la vita è parte di una grande processo ritmico di creazione e distruzione, morte e rinascita, e il ballo di 
Shiva simboleggia questo movimento eterno di vita e morte che continua in cicli infiniti, attraverso la relazione e il gesto performativo postumano.

\section{Digital Queerism}

Da Tanaka a Bjork, la ricerca artistica ed espressiva al femminile per prima ha intuito e declinato le possibilità di ibridazione con il mondo ipermediale e con la dimensione naturale, in una parola con il non-umano, l'altro-da-sé, attraverso il gesto, i toni accoglienti dello scambio, dell'apertura, della dissoluzione dell'antropocentrismo. In questo solco si collocano ricerche ed esperienze di ridefinizione del sé attraverso la relazione con la tecnosfera e le sottoculture da essa derivate, soprattutto in chiave corporea e di genere, nelle affermazioni di inedite modalità dell'essere che spesso compaiono e mutano in diretta, davanti ad uno schermo connesso, in fronte ad un pubblico presente virtualmente. Flusso di molteplicità disordinate, amalgama, improvvisazioni intuitive, bricolage post-culturale, procedure alchemiche; tutte volte a sancire la pluralità dell'esistenza attraverso l'azione espressiva, al di là di confini, categorie, definizioni. Penso al progetto Isadora, residenza digitale di esplorazione e adattamento sul social network TikTok, realizzata dalla coreografa Giselda Ranieri; alle pratiche di rilettura e ricontestualizzazione delle estetiche e dei linguaggi corporei provenienti dalle sottoculture degli anni Duemila - mixate con altre tradizioni e contesti - messe in scena, fra le altre, da Mara Oscar Cassiani, Anne Imhof, Dana Michel; alla conquista di ambienti virtuali attuata dall'avatar LaTurbo Avedon; alla diffusione di nuove maschere virtuali come filtri per Instagram realizzata da Kamilia Kard, Massiel Leza, da indossare digitalmente dal vivo.

Quanto dice, d'altronde, di noi una raffigurazione multimediale oggi? In che modo le tecnologie contemporanee influiscono sulla rappresentazione del sé, sul ritratto - figurativo e narrativo - che ognuno presenta alla propria comunità di riferimento? E sulla propria identità, può essere quest'ultima considerata come postumana, amplificata dalla relazione continua coi media? Nelle mani delle artiste e degli artisti, i media possono non solo estendere all'arte le potenzialità del virtuale e del digitale, ma anche donare la chance di oltrepassare i confini corporei materiali, in direzione spirituale e questa estensione, che diventa energia tracimante i confini di spazio e tempo, viene riattivata ancora diversamente da progetti tea- 
trali e performativi, opere d'arte totali, le quali in un caleidoscopio di luci, corpi, messaggi, offrono uno spettacolo di potente coinvolgimento. Invitando ciascuno di noi a viversi come spettacolo, come scrisse alla fine degli anni Sessanta l'artista Paolo Scheggi ${ }^{14}$.

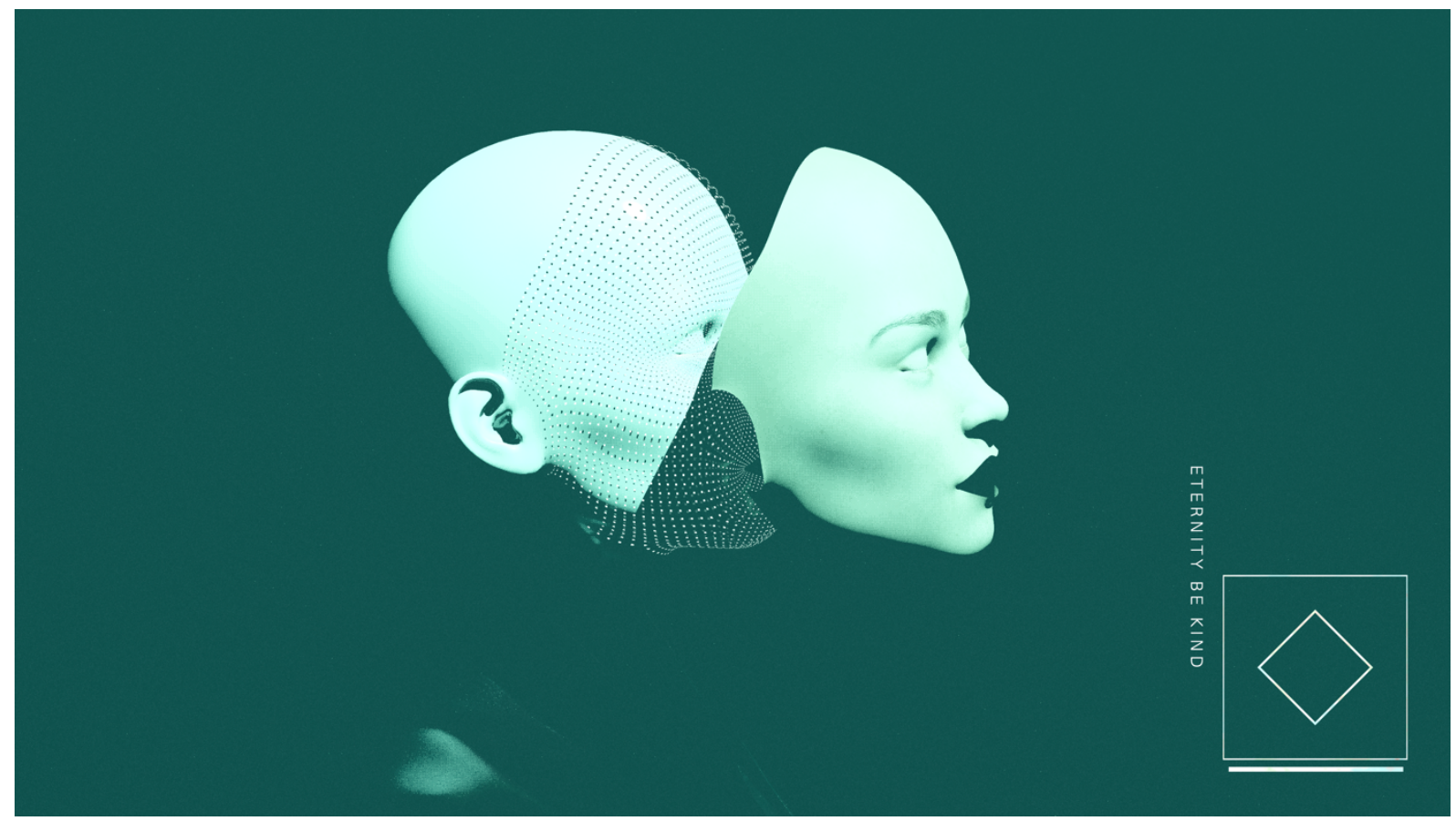

Fig. 03. Myriam Bleau \& LaTurbo Avedon, Eternity Be Kind, 2020. Immagine dal materiale promozionale della performance

\section{Chi ha paura del live streaming?}

L'Era pandemica ha visto crescere divisioni e confusioni rispetto alle possibilità rappresentate dalla condivisione di contenuti in streaming. Sistema di trasmissione dati attraverso una rete telematica, questo flusso multimediale può essere trasmesso in diretta (live) o su richiesta (on demand). Dal punto di vista di chi fa e studia lo spettacolo dal vivo, lo streaming on demand non ha nessuna sfumatura di liveness, né per l'autore né per lo spettatore. La questione si complica invece se andiamo ad analizzare un live streaming: dove l'emissione del flusso multimediale avviene sempre in tempo reale, i contenuti, i dati trasmessi possono essere stati pre-registrati. Ovvero: non è totalmente eseguito, trasmesso e fruito in real time tutto quel che viene denominato live. La confusione rischia di sminuire l'esecuzione e la fruizione in diretta online, oltre ad alimentare la convinzione sbagliata che queste esibizioni siano da considerarsi artisticamente, teatralmen-

${ }^{14}$ P. Scheggi, La città come tempo di spettacolo, in «Casabella» vol. XXXIII, agosto-settembre 1969, pp. 94-95. 
te irrilevanti. Si tratta di strategie e differenze che sottendono linguaggi e poetiche precise da non confondere, nè sottovalutare: molti performer compiono una precisa scelta estetica non esibendosi in live streaming, a causa delle difficoltà tecniche di questa operazione e per la inevitabile compressione della qualità dei dati; per la ricezione spesso insufficiente che il pubblico riceverebbe, o perché preferiscono optare per un formato chiuso: registrare e poi mostrare un video postprodotto, più simile a un documentario o a un film. Altri invece trovano nella rude immediatezza di questa con-presenza da remoto una possibilità pervasiva per raggiungere, invadere e inglobare la vita quotidiana di ognuno; la modalità più adatta a conservare un contatto, seppur virtuale, nonostante le distanze e le diversità spaziali, con il pubblico, cui far vivere un'esperienza now or never.

La consapevolezza rispetto a cosa si andrà a fruire online e come lo si vuole presentare diviene fondamentale, di fronte all'espansione e alla saturazione del settore, per una scelta critica. Di seguito alcuni esempi di elaborati e produzioni che nel live streaming hanno trovato una dimensione estetica e drammaturgica fondamentale allo sviluppo dell'opera.

Nella vita quotidiana, gli esseri umani assemblano una pletora di segnali informativi in unità gestibili e li riconoscono, per esempio, come un individuo, un oggetto o un certo scenario. Quando si guarda una cascata da lontano, invece, questa sembra mantenere forme statiche; tuttavia, quando si zooma sugli stessi oggetti a livello microscopico, si nota che sono costituiti da movimenti incessanti come mareggiate, onde, vortici e correnti incrociate. In accordo con la fisica quantistica, il maestro della scena artistica d'avanguardia giapponese Hiroaki Umeda ipotizza che questi lotti di informazioni astratte siano semplicemente il sistema di credenze umane:

Quando si ha fiducia nella fattualità di un oggetto lo si chiama reale, e quando questa fiducia è leggermente minata lo si rinomina come virtuale. [...] Se tutti i movimenti cinetici sono componenti estetici uguali della coreografia, allora potrebbe esistere un pezzo coreografico senza esseri umani. Inoltre, se l'obiettivo principale dell'artista sta nel fornire al pubblico la sensazione fisica pre-linguistica e pre-emozionale, che alternativamente potrebbe essere chiamata impulso, questa esperienza può essere generata possibilmente senza il mezzo del corpo in movimento. ${ }^{15}$

\footnotetext{
${ }^{15}$ E. Roche, Hiroaki Umeda: Artist Thoughts - Composing Holistic Sensations, dal sito dell'artista Hiroaki Umeda, mia traduzione: http://hiroakiumeda.com/artist.html (ultimo accesso 10/11/2021).
} 
Se tutto è dissolto in atomi e protoni, non c'è molta differenza tra esseri umani e oggetti, e non c'è molta certezza tra individualità e interezza. E quando il mondo viene osservato attraverso questa nuova lente adottata, alla fine emerge un mondo eticamente più umile, non antropocentrico e post-umanista. Sulla base di questo concetto creativo, in Intensional Particle, Umeda reinterpreta le particelle nello spazio non come molecole statiche ma piuttosto come particelle attive; e visualizza, nello spazio, la forza intenzionale che le particelle nascondono.

Il suo lavoro è riconosciuto per la sua metodologia artistica altamente olistica con un forte baround digitale, che considera non solo elementi fisici come la danza ma anche componenti ottiche, personali, sensoriali e, soprattutto, spazio-temporali come parte della coreografia: sembra incarnare perfettamente il concetto di Barad di intra-azione performativa tra esseri umani e agenzia meccanica. Al contrario, le culture digitali sono culture performative. La performatività nelle sue dimensioni eterogenee non può permettersi di ignorare le forze e gli effetti agenziali delle tecnologie digitali e i loro intrecci con i corpi umani. E così fa Umeda.

Dopo un debutto offline nel 2015, in tempi pandemici il suo lavoro Intentional Particle ha trovato una versione play remote live online. La sfida è diventata quella di formare le migliori condizioni per lo spettatore, a casa sua: come se venisse quantisticamente teletrasportata, la silhouette di Umeda appare in azione e trasmette il flusso energetico ai corpi remoti. Anche se attraverso lo specchio nero, il pubblico sarà immerso in una realtà digitale satura di "stabilità instabile", innescata da un flusso di stimoli ottici e sonar. Merleau Ponty direbbe che "ll corpo è il nostro mezzo generale per avere un mondo» ${ }^{16}$ dopo tutto.

Il collettivo di fama mondiale Rhizomatiks sta mettendo alla prova il web, presentando performance e sistemi sperimentali online, e affrontando queste situazioni da diverse angolazioni: le collaborazioni con una gamma diversificata di performer internazionali, per esempio, hanno creato nuove visioni del corpo entangled con la tecnologia, mentre le sperimentazioni con scienziati e ricercatori, tra cui neuroscienziati e astronomi, hanno permesso a ciò che è invisibile di apparire nelle composizioni multimediali del gruppo artistico giapponese, attraverso la visualizzazione dei dati.

\footnotetext{
${ }^{16}$ M. Merleau-Ponty, Fenomenologia della percezione [1945], Bompiani, Milano 2003, p. 292.
} 
Nel mondo mercuriale, complesso e ambiguo di oggi, i corpi oscillano tra il virtuale e il reale. Integrando l'informatica, la codifica e le tecnologie ingegneristiche con la grande varietà di comunicazione nascosta nella società moderna e la fisicità umana, compresa la virtualità, Rhizomatiks ha prodotto molte opere che rivelano nuovi aspetti e intuizioni, esplorando possibilità di con-presenza in real time fra spazio fisico e virtuale, online. In uno dei nuovi lavori di installazione, multiplex, le performance fisiche generate da un visual design e una programmazione esemplari, attraverso collaborazioni con artisti come ELEVENPLAY e Perfume, si trasformano in un mezzo che connette il pubblico con il movimento e lo spazio, dando vita a una versione finora sconosciuta del corpo. Qui, le proiezioni creano un ambiente immersivo con immagini perfettamente mescolate e un layout ottimizzato che aumenta il senso di realismo. Viceversa, lo spazio reale è integrato in uno virtuale online, spostando la prospettiva dello spettatore e permettendo esperienze implementate, ultra umane.

La ricerca dei Rhizomatiks si è sempre concentrata sui linguaggi performativi multimediali e sulla loro evoluzione live media show. I set e le performance dal vivo continuano a contribuire alla costruzione di un'immagine postumana dei corpi in scena, connettendo software, hardware, gesti, musica, dimensioni naturali e artificiali.

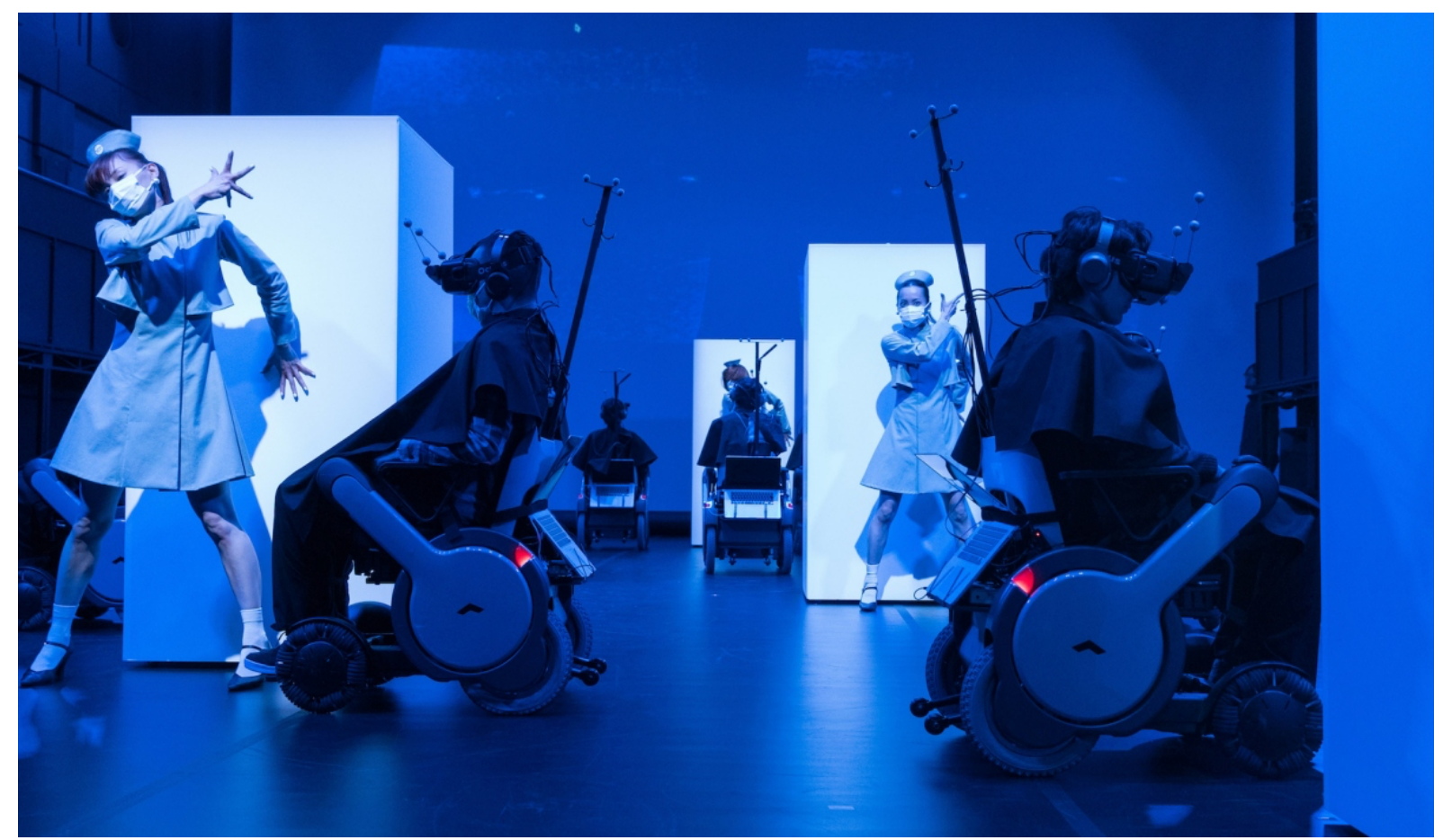

Fig. 04. ELEVENPLAY×Rhizomatiks Research, borders, 2021. Foto: Muryo Homma 
Hanno recentemente ri-presentato borders, un pezzo di danza precedentemente sviluppato con ELEVENPLAY e MIKIKO nel 2015. Sul palco, in presenza fisica "in-real-life", borders prevede un ambiente dove il pubblico può mantenere la distanza sociale per guardare una performance di danza dal vivo integrata con tecnologie di mobilità e realtà mista da più punti di vista. Inoltre, il progetto offre un nuovo modo di godersi lo spettacolo consentendo l'accesso online e, soprattutto, fornendo un'esperienza visiva unica come una vista a volo d'uccello dell'intera sede e la schermata operativa del software di controllo, che non può essere vista nell'esperienza in loco.

Per il coreografo Choy Ka Fai i media digitali possono essere alleati nella riscoperta e reinterpretazione della spiritualità. Originario di Singapore e di base a Berlino, è da tempo affascinato dal corpo umano come strumento per esplorare esperienze mistiche e idee coreografiche postumane, e dalle potenzialità extracorporee, sublimi, indotte dall'esperienza multimediale. Il suo lavoro si basa sulla creazione e sul dialogo con avatar e intelligenze artificiali «metà umani e metà qualcos'altro», mescolando nuove tecnologie con antichi rituali e intrecciando gli stati alterati della spiritualità con speculazioni sul corpo postumano.

Per Cosmic Wander, il suo ultimo progetto in corso, l'artista si è avventurato attraverso l'Asia per studiare le pratiche spirituali e le danze sciamaniche di diversi popoli e comunità. Durante i suoi viaggi ha incontrato oltre 50 sciamani e ha filmato straordinarie esperienze: una ricerca ispirata alle pratiche osservate a Singapore, in Indonesia, in Siberia, a Taiwan e in Vietnam. Al Tanz im August, festival internazionale di danza contemporanea di Berlino, nell'agosto 2021 ha presentato anche la performance Postcolonial Spirits: un rituale di trance accompagnato da canzoni su miti locali, battute politiche e poesie islamiche. Ispirato al patrimonio multidimensionale della danza popolare indonesiana Dolalak che si è continuamente adattato alla cultura pop e ai media, mescolando le imitazioni dei soldati coloniali olandesi alle feste cosplay degli anni ‘30, Postcolonial Spirits ha visto in scena un'esperienza di telepresenza con performers collegati via livestream da Giava, e il performer Vincent Riebeek fisicamente sul palco di Berlino. "Cosmic Wander" prevede anche una restituzione come esperienza immersiva in VR: nella Blue Sky Academy è possibile interagire con avatar di sciamani e danzatori, le cui sembianze richiamano sia la fisicità delle persone intervistate e filmate da Kafai 
(rilevata tramite sensori e body scanner) sia le fattezze delle divinità con cui questi personaggi sono interconnessi.

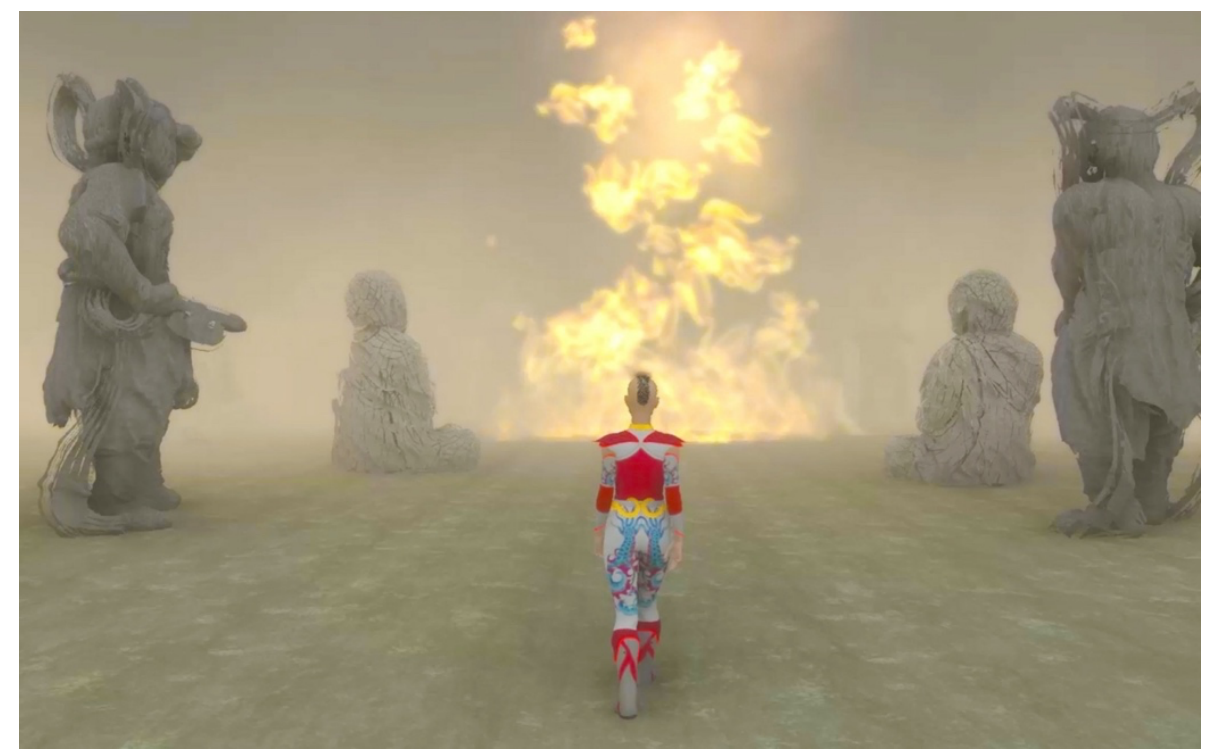

Fig. 05. Choy Ka Fai, Postcolonial Spirits, 2021. Still da video della performance

Antibody è la nuova produzione teatrale del coreografo inglese Alexander Whitley, un'esperienza di danza online che esplora la forma biologica del corpo umano e la spinta tecnologica a trascenderla. Tre ballerini sono isolati l'uno dall'altro in un ambiente interattivo collegato attraverso uno spazio centrale di informazioni puramente digitali; strati visivamente accattivanti di grafica 3D sensibili al movimento, creati da Uncharted Limbo Collective, agiranno per rappresentare le forme simboliche e virtuali della nostra esistenza, in contrasto con il mondo fisico dei nostri corpi. Tra questi due strati di azione e rappresentazione si svolge una doppia narrazione che esplora le tensioni tra mente e corpo, contenimento e connessione e il desiderio di essere unici e parte di qualcosa di più grande. Nell'offerta digitale, i ballerini dal vivo sono sostituiti da versioni olografiche di loro stessi, create utilizzando una tecnologia di cattura volumetrica all'avanguardia. Lo spettacolo, concepito per la fruizione dal vivo sia IRL che in streaming, ha debuttato alla DanceEast nell'ottobre 2021. La produzione verrà poi filmata utilizzando il green-screen e la tecnologia volumetrica e da diverse angolazioni della telecamera, per permettere alla pièce di essere vissuta digitalmente in diversi formati. 


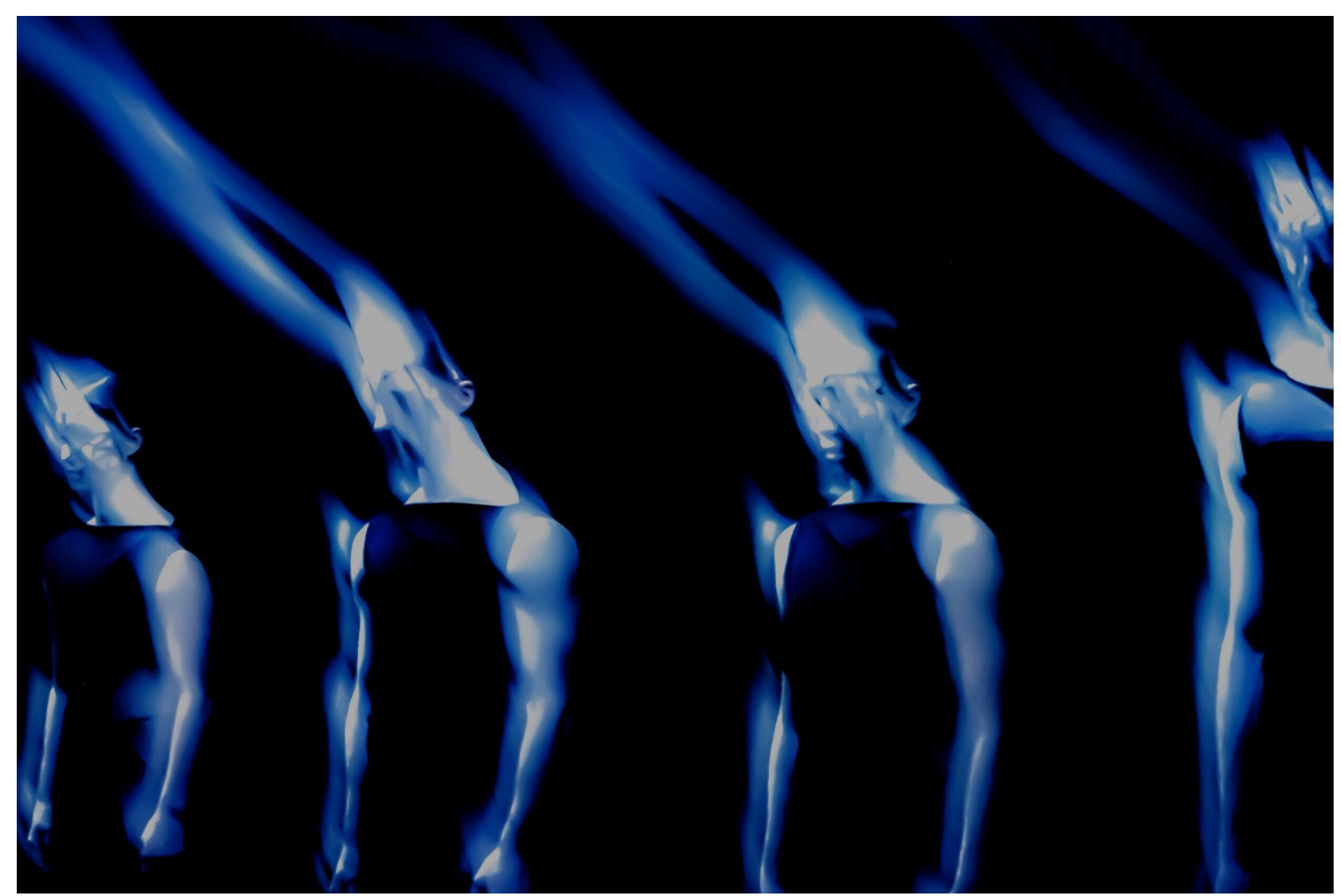

Fig. 06. Alexander Whitley, Antibody, 2021. Immagine dal sito dell'artista

\section{Game Feel}

L'avvento del Metaverso rappresenta un cambiamento fondamentale nell'odierna nozione di presenza digitale. Motori di grafica 3D in tempo reale come Unity e Unreal Engine sono sempre più spesso utilizzati per la creazione di esperienze espositive e performative virtuali, poiché offrono una serie di funzionalità personalizzabili in grado di costruire un'infinita varietà di esperienze avanzate, da fruire in real time in maniera semplice e affascinante. La user experience of art (UXA) rimodella e rivaluta le strategie digitali con l'avvento di questi ambienti virtuali avanzati, e colloca l'emergere dell'arte "Metaverso-nativa" in campi adiacenti all'arte come il gioco, la blockchain, il cinema e l'architettura. Fra gli ambienti in cui la liveness multimediale - della fruizione e della generazione dei contenuti - ha da sempre giocato un ruolo fondamentale per lo sviluppo di una esperienza estetica - e ludica - i videogiochi rappresentano ora per le performing arts un terreno da esplorare. Dopo lo storico intervento di Franco ed Eva Mattes, gli esempi di sperimentazione artistica e performativa all'interno delle simulazioni e delle gamers community continuano a crescere; non solo, molte di queste piattaforme stanno svilup- 
pando modalità di fruizione art adjacent, come Fortnite Creative, per agevolare le derive sperimentali e creative degli utenti.

Gestito da un collettivo artistico di base a Manila, a partire dal 2019 Club Matryoshka è stato uno dei music club virtuali più famosi fuori e dentro il Metaverso, situato su un server privato di Minecraft. Oltre ai rave party, negli ultimi tempi la piattaforma di gioco ha ospitato numerosi eventi live: le edizioni virtuali di festival come il Coachella, ma anche spettacoli di teatro come The House of Sorrow del regista coreano Jisun Kim, esposizioni e progetti di residenza artistica come quelli promossi dalla Ender Gallery. Fino agli happenings di Tania Candiani: per il progetto El tiempo es otro rio, l'artista messicana ha organizzato dei raduni di protesta sulle piazze virtuali di Minecraft, Animal Crossing, Sims e Second Life.

Roblox, usato per lo più dai teens, è la piattaforma di gioco e connessione scelta da Kamilia Kard per la sua performance partecipativa Dance Dance Dance, in cui gangs estemporanee di avatar vengono coinvolte in balli di gruppo sincronizzati (con coreografie prese da librerie mocap, videogiochi famosi, videoclip, TikTok, e dalla danza contemporanea) all'interno di un ambiente virtuale programmato e disegnato dall'artista.

L'universo gaming induce emozioni profonde nell'utente, genera meta-sensazioni di coinvolgimento radicale: il game feeling è un'esperienza estetica sofisticata, interattiva, controversa, estrema, economicamente sempre più potente, perfetta per lo sviluppo della sperimentazione artistica contemporanea. Una dimensione onirica per l'utente, da esperire in tempo reale.

\section{Al + Music}

Mentre il concetto di musica composta da macchine risale al 1800 (la pioniera dell'informatica Ada Lovelace fu una delle prime a scrivere sull'argomento), la fantasia è diventata realtà nell'ultimo decennio, con musicisti come Francois Pachet che hanno creato interi album scritti dall'Al.

Nel suo tentativo di trovare il suono di "ora”, la musicista elettronica Holly Herndon trova conforto nel potere del canto collettivo e nella rivendicazione del potere umano sull'intelligenza artificiale. Nella composizione dell'album e nell'esecuzione della perfor- 
mance live PROTO, Herndon è accompagnata sul palco, dal vivo, da un esteso ensemble vocale, da un'esperienza audiovisiva di nuova concezione e da un sistema neurale di intelligenza artificiale operativo in real time chiamata Spawn. Sviluppata con l'artista e filosofo Mathew Dryhurst e lo sviluppatore di ensemble Jules LaPlace, la "baby Al" di Herndon ha imparato il suo vocabolario sonoro attraverso sessioni in studio e training vocale pubblico. I suoni che produce provengono dalla sua immaginazione, mentre cerca di imitare come Herndon e i suoi collaboratori suonerebbero cantandoli. Come spiega Herndon, "Spawn è un membro dell'ensemble come qualsiasi altro, e contribuisce al lavoro con idee alla pari. Questo è un rapporto speciale, e simbolico, da sviluppare con la materia. Gli umani replicano i processi digitali; le intelligenze digitali replicano gli umani» ${ }^{17}$.

La relazione fra Herndon e Spawn segna anche una nuova direzione per l'adozione di processi di apprendimento automatico nella musica in generale. «La maggior parte dei progetti di musica Al si sono concentrati sulla creazione di forme musicali automatizzate, piuttosto che sul cambiamento delle possibilità del modo in cui la musica suona. Troviamo quest'ultimo approccio molto più convincente - non si tratta di sostituire un umano, ma piuttosto di osservare e aumentare le abitudini umane $»^{18}$. Per Herndon e i suoi collaboratori, questo mutualismo con I'IA è un gesto radicale ma essenziale, in particolare quando viene utilizzato per reimmaginare la nozione storica del canto collettivo come strumento di sopravvivenza ${ }^{19}$.

\section{Conclusioni}

Le suggestioni filosofiche interpretative, poetiche proposte da Barad, e qui mutuate, sembrano voler narrare la realtà come continua tessitura, fluida, di relazioni, connessioni, scambi, gesti a livello particellare. Uno scambio energetico, costituente della identità stessa delle entità agenti, che avviene costantemente, a qualsiasi e fra qualunque livello di esistenza, come insegna la fisica quantistica. La relazione avviene quindi in ogni momento, è sempre viva e attiva, fra diverse specie, mondi, tipologie.

\footnotetext{
${ }^{17}$ H. Herndon, B. Seaton, Brit Seaton talks to Holly Herndon about community, collective singing and Al music, [traduzione mia], https://www.barbican.org.uk/holly-herndon-proto (ultimo accesso 10/11/2021).

18 lbidem, [traduzione mia].

${ }^{19} \mathrm{H}$. Herndon, M. Dryhurst, Listening to the Voice of \#Al, with Holly Herndon and Mat Dryhurst, Sonar+D Talk, 2019, https://www.youtube.com/watch?v=k67IKMXzD7A (ultimo accesso 10/11/2021).
} 
Le cangianti rappresentazioni digitali sembrano così ricreare più efficacemente la dimensione viva, metamorfica, evolutiva dell'esistenza così come descritta da Barad; nella relazione con l'umano poi aggiungono possibilità di esperienze aumentate, inedite, contraddistinte da una progressiva dematerializzazione ma soprattutto da una costante interconnessione, che permette una presenza virtuale estesa, stratificata.

Una realtà come quella descritta pare forse di difficile comprensione, e sembra necessitare di una struttura esplicativa empirica; oppure, attraverso l'intuizione e l'espressività poetica, può costituirsi come suggestione, forse non per la comprensione ma per una maggiore empatia con i mutamenti in atto che attraversano la contemporaneità.

II gesto artistico, nei secoli, ha sempre rappresentato una forma creativa e culturale devota all'avvicinamento e alla "costruzione di ponti" verso l'ignoto, un atto di traduzione dell'incomprensibile in rituale più facilmente processabile. Le opere e gli artisti qui presentati, seppur rappresentino una campionatura non esaustiva della produzione nel settore delle live media performance, possono allora essere intesi come casi di studio emblematici delle teorie di Barad (e non solo); esempi simbolici contemporanei, metafore utili a colmare le distanze e capaci invece di rappresentare poeticamente la fluidità dell'esistenza - un'esistenza che comprende e compenetra la coalescenza fra società polarizzate, strutture robotiche e algoritmiche ed ecosistemi naturali - la sua continua dinamica relazionale, i vari livelli e le varie possibilità di presenza. Nell'intento di sostenere la decolonizzazione del pensiero, anche artistico, oltre concetti e categorie obsolete.

\section{Riferimenti Bibliografici}

P. Auslander, At the Listening Post, or, do machines perform?, in «International Journal of Performance Arts and Digital Media», vol. 1, n. 1, 2005, pp. 5-10.

A. Balzola, A. Monteverdi, Le arti multimediali digitali. Storia, tecniche, linguaggi, etiche ed estetiche del nuovo millennio, Garzanti, Milano 2004.

K. Barad, Re(con)figuring Space, Time, and Matter, in M. DeKoven (a cura di) Feminist Locations: Global and Local, Theory and Practice, Rutgers University Press, New Brunswick, 2001.

K. Barad, Posthumanist Performativity: Toward an Understanding of How Matter Comes to Matter, in «Signs: Journal of Women in Culture and Society», vol. 28, n. 3, 2003, pp. 801-831. 
K. Barad, Meeting the Universe Halfway: Quantum Physics and the Entanglement of Matter and Meaning, Duke University Press, Durham 2007.

K. Barad, What is the Measure of Nothingness? Infinity, Virtuality, Justice / Was ist das Maß des Nichts? Unendlichkeit, Virtualität, Gerechtigkeit, in dOCUMENTA (13): 100 Notes 100 Thoughts / 100 Notizen - 100 Gedanken, Hatje Cantz Verlag, Ostfildern 2012.

K. Barad, On Touching -- The Inhuman That Therefore I Am, in «Differences: A Journal of Feminist Cultural Studies», vol. 23, n. 3, 2012, pp. 206-223.

S. Bay-Cheng, C. Kattenbelt, A. Lavender, Mapping Intermediality in Performance, Amsterdam University Press, Amsterdam 2010.

W. Benjamin, Aura e choc. Saggi sulla teoria dei media, Einaudi, Torino 2012.

T. Beyes, M. Leeker, I. Schipper, Performing the Digital. Performance Studies and Performances in Digital Cultures, Transcript-Verlag, Bielefeld 2016.

J. Bolter, R. Grusin, Remediation: Understanding New Media, MIT Press, Cambridge 1998.

N. Bourriaud, Estetica Relazionale, Postmediabooks, Milano 1998.

R. Braidotti, The Posthuman, Polity Press, Cambridge 2013.

J. Bridle, Nuova Era Oscura, Nero, Roma 2019.

L. Cornell, E. Halter (a cura di), Mass Effect: Art and the Internet in the Twenty-First Century, MIT Press, Cambridge 2015

M. Costa, L'estetica dei media. Avanguardie e tecnologia, Castelvecchi, Roma 1999.

S. Dixon, Digital Performance: A History of New Media in Theater, Dance, Performance Art, and Installation, MIT Press, Cambridge 2007.

P. Eckersall, H. Grehan, E. Scheer, New Media Dramaturgy. Performance, Media and NewMaterialism, Palgrave Macmillan, Londra 2017.

D. Haraway, Manifesto Cyborg. Donne, tecnologie e biopolitiche del corpo, Feltrinelli, Milano 2018.

K. Kard (a cura di), Alpha Plus: anthology of digital art, Editorial Vortex, 2017.

L. Manovich, Il Linguaggio dei Nuovi Media, Edizioni Olivares, Milano 2002.

G. Matteucci, Elementi per una estetica del contemporaneo, Bononia University Press, Bologna 2018.

M. Merleau-Ponty, Fenomenologia della percezione, Bompiani, Milano 2003.

A. Monteverdi, Leggere uno spettacolo multimediale. La nuova scena tra video mapping, interaction design e Intelligenza Artificiale, Dino Audino Editore, Roma 2020. 
S. O’Reilly, Il corpo nell'arte contemporanea, Einaudi, Torino 2009.

C. Paul, Digital Art (3rd ed.), Thames \& Hudson, London 2015.

R. Peppeler, The Posthuman Condition: Consciousness Beyond the Brain, Intellect, Bristol 2003.

M. Perniola, L'estetica contemporanea, il Mulino, Bologna 2011.

E. Povinelli, The Ends of Humans: Anthropocene, Autonomism, Antagonism, and the Illusions of Our Epoch, in «The South Atlantic Quarterly», vol. 116, n. 2, 2017, pp. 293-310.

D. Quaranta, Beyond New Media Art, Link Editions, Brescia 2013.

F.J. Ricardo, The Engagement Aesthetic. Experiencing New Media Art through Critique, Bloomsbury Academic, London 2013.

C. Salter, Entangled: Technology and the Transformation of Performance, MIT Press, Cambridge 2010.

P. Scheggi, La città come tempo di spettacolo, in "Casabella» vol. XXXIII, agosto-settembre 1969, pp. 94-95

H. Steyerl, Duty Free Art: Art in the Age of Planetary Civil War, Verso books, London 2017.

G. A. Voorhees, I Play Therefore I Am: Sid Meier's Civilization, Turn-Based Strategy Games and the Cogito, in «Games and Culture», vol. 4, n. 3, 2009, pp. 254-275.

\section{Sitografia}

Intervista a Holly Herndon: https://www.barbican.org.uk/holly-herndon-proto (ultimo accesso $10 / 11 / 2021)$.

Holly Herndon in conversazione con Mat Dryhurst al Sonar+D Talk 2019:

https://www.youtube.com/c/Sonarplusd (ultimo accesso 11/11/2021).

Sito dell'artista Hiroaki Umeda: http://hiroakiumeda.com/artist.html (ultimo accesso 10/11/2021)

Intervista a Karen Barad: https://www.sovrapposizioni.com/blog/la-materia-sente-conversasoffre-desidera-anela-e-ricorda-intervista-a-karen-barad-prima-parte (ultimo accesso $10 / 11 / 2021)$

\section{Biografia dell'autore/ Author's biography}

Federica Patti è storica dell'arte, docente e curatrice indipendente, la cui ricerca si concentra sulle arti multimediali digitali, su progetti interattivi e partecipativi, sulle live media performance con una propensione all'apertura e alla contaminazione verso pubblici e spazi non convenzionali. Vincitrice 
della nona edizione dell'Italian Council nel 2020 con il progetto "La performatività posthuman. Una definizione transdisciplinare della live media performance" con il sostegno di Direzione Generale Creatività Contemporanea. Attualmente collabora con MAMbo - Museo d'Arte Moderna Bologna, CUBO - Centro Unipol Bologna e Romaeuropa Festival nell'ideazione e realizzazione di mostre e attività educative legate ai temi di arte, scienza, nuove tecnologie. È membro del collettivo LaRete Art Projects e di IKT - International Association of Curators of Contemporary Art. Scrive per le riviste Artribune magazine e Creativeapplication.net.

Federica Patti is an independent curator, lecturer and art critic. Her research focuses on contemporary experimental art movements, with a particular interest in multimedia, live media, emerging artists, interactive and participatory projects. Winner of the ninth edition of the Italian Council in 2021, with the project "On Posthuman Performativity. For a trans-disciplinary definition of live media performance" supported by the Directorate-General for Contemporary Creativity by the Italian Ministry of Cultural Heritage and Activities and Tourism under the Italian Council program (2020), she actively collaborates with several institutions, galleries and festivals (MAMbo - Museo d'Arte Moderna Bologna, CUBO - Centro Unipol Bologna, Romaeuropa Festival among others) on the presentation and development of her research topics curating exhibitions, screening, talks and workshop. She is part of LaRete Art Projects curatorial collective and of IKT (International Association of Curators of Contemporary Art). Since 2013 she has been a frequent contributor for Artribune magazine and Creativeapplication.net. 